\title{
Stochastic Model of Three Products Inventory System Assuming Seasonal Demand Following Exponential Distribution
}

\author{
D.Kanagajothi, S. Srinivasaragavan
}

\begin{abstract}
In this paper we discuss the production and sales of three different products $A, B$ and $C$ with maximum production size as $k$ sets of them. The production of the products $A, B$ and $C$ are done in order. When one set of $A, B$, and $C$ is produced, the production of the next set starts. The sales of the sets begin when $k$ sets are produced or when the season for the products starts whichever occurs first.The production times of the products are assumed to have general distributions and the season starts in an exponential time. The double Laplace Transform of production and sale time and their means are obtained. Numerical examples are presented.
\end{abstract}

Key words: Inventory systems, production models, stochastic models.

Mathematics 2010 Subject Classification: 90B05, 90B30, $91 B 70$.

\section{INTRODUCTION}

A lot of companies use to inventory system, uplift their business. Various types of triplet products are manufactured to sell. The productions of three products inventory system have several examples. Tube light manufacturing companies produce light, chock and holder. Stabilizer companies produce engine part of the body, filter and Ultraviolet. Tiles manufacturing companies produce three different tiles for living room, kitchen and bathroom. A number of researches are done on single commodity inventory systems of $(\mathrm{s}, \mathrm{S})$ type and it is found that inventory systems and their relation to the inventory of triple products are impactful by seasonal demand. Several researchers studied single commodity inventory systems of (s, S) type. Daniel, J.K. and Ramanarayanan, $R[1,2]$ discussed an $(S, s)$ inventory system with two servers and rest period to the server. Murthy, S. and Ramanarayanan, $\mathrm{R}$ [3, 4] considered several (s, S) inventory system and they studied one ordering and two ordering levels inventory system with many property. Parvathi $S$ et. al $[5,6]$ analyzed one product inventory system with seasonal product and sales. Usha, K., analysis of inventory system with sales time depending on production time. Usha, K., Nithyapriya, N. and Ramanarayanan, R [8] inventory system with defective suppliers and financial support for customer. In this study inventory systems and their related inventory of triple products subject to seasonal demand is presented. The

Revised Manuscript Received on September 10, 2019.

D.Kanagajothi, Department of Mathematics, Vel Tech Rangarajan Dr.Sagunthala R\&D Institute of Science and Technology, Avadi, Chennai, Tamil Nadu, India.

(email: kanagajothi82@gmail.com)

S. Srinivasaragavan, Department of Mathematics, Vel Tech Rangarajan Dr.Sagunthala R\&D Institute of Science and Technology, Avadi, Chennai, Tamil Nadu, India.

(email: kanagajothi82@gmail.com). Nithyapriya, N. and Ramanarayanan, R [7] Stochastic

double Laplace Transform of production and sale time and their means are obtained. Numerical examples are presented.

\section{MODEL}

The following are the assumptions of the model

(i) Three different products $A, B$ and $C$ are produced by the company. But only one is produced at a given time. The production of the products $A, B$ and $C$ are done in order. Only when one set of $A, B$ and $C$ is produced, the production of the next set starts. The production times of $A, B$ and $C$ are random variables $X, Y$ and $Z$ respectively. The production duration $X$ of product $A$ has c.d.f $H_{X}($.$) and p.d.f h_{X}($.$) ; the$ production duration $Y$ of product $B$ has c.d.f $H_{Y}($.$) and p.d.f$ $h_{Y}($.$) and the production time Z$ of product $C$ has c.d.f $H_{Z}($.$) and p.d.f h_{Z}($.$) . The production time X+Y+Z$ of one unit of triplet has c.d.f $\mathrm{H}$ (.)..

(ii) The season with demand for the units starts with an exponential time with parameter $\lambda$ from starting time zero.

(iii) When $\mathrm{k}$ number of units of triplet products are produced time of sales begin. Sales time also begins when demand for the products arises.

(iv) The units are sold one by one and the selling time of a triplet is random variable with c.d.f $S($.$) with p.d.f s (.).$ The c.d.f of selling times of individual products $A, B$ and $C$ $\operatorname{are} S_{A}(),. S_{B}($.$) and S_{C}($.$) with p.d.f s_{A}(),. s_{B}($.$) and s_{C}($. respectively.

\section{ANALYSIS \& RESULTS}

It is noted that the probability of $n$ unit is produced in the interval $(0, t)$ is $H_{n}(t)-H_{n+1}(t)$ for $n \geq 0$ where $H_{n}(\mathrm{t})$ is $n$ fold c.d.f convolution of $H(t)$ and $H_{n}(t)$ is the c.d.f of $\sum_{i=1}^{n}\left(X_{i}+Y_{i}+Z_{i}\right)$. During the period $(0, t)$, when the $n^{\text {th }}$ triplet is completed by the company at time $x<t$, during the remaining period $(x, t)$, the production part done by the company may be as follows:

a) The production time for product $A$ is not over or

b) The product $A$ is produced, but the product $B$ is not produced or

c) The products $A$ and $B$ are produced, but the product $C$ is not produced

before time t. Their respective probabilities are given below. Let $h_{n}$ (x) be $n$ fold convolution of $h(x)$ with itself, where $h(x)$ is the p.d.f of $\quad X+Y+Z$ and $h_{n}(\mathrm{x})$ is the p.d.f of $\sum_{i=1}^{n}\left(X_{i}+Y_{i}+Z_{i}\right)$. Then the probability of $\mathrm{n}$ triplet units is produced in $(0, t)$ and the $(n+1)$ th production of $A$ is not completed before $t$ is Equation 3.1 


$$
\begin{gathered}
\mathrm{P}\left[\sum_{i=1}^{n}\left(X_{i}+Y_{i}+Z_{i}\right)+X_{n+1}<t<\sum_{i=1}^{n}\left(X_{i}+Y_{i}+Z_{i}\right)\right] \\
=\int_{0}^{t} h_{n}(x) \bar{H}_{X}(t-x) d x
\end{gathered}
$$

The probability of $n$ triplet units is produced, but one production of $A$ is over before $t$, but production of $B$ is not over before $t$ is

$$
\begin{gathered}
P\left[\sum_{i=1}^{n}\left(X_{i}+Y_{i}+Z_{i}\right)+X_{n+1}+Y_{n+1}<t<\sum_{i=1}^{n}\left(X_{i}+Y_{i}+Z_{i}\right)+X_{n+1}+Y_{n+1}\right] \\
=\int_{0}^{t} h_{n}(x) \int_{0}^{t-x} h_{X}(u) \bar{H}_{Y}(t-x-u) d u d x
\end{gathered}
$$

The probability of $\mathrm{n}$ triplet units is produced. But the production of $A$ and $\mathrm{B}$ are over before $\mathrm{t}$, but production of $\mathrm{C}$ is not over before $t$ is Equation 3.2

$$
\begin{aligned}
P\left[\sum _ { i = 1 } ^ { n } \left(X_{i}+Y_{i}\right.\right. & \left.+Z_{i}\right)+X_{n+1}+Y_{n+1}+Z_{n+1}<t \\
& \left.<\sum_{i=1}^{n}\left(X_{i}+Y_{i}+Z_{i}\right)+X_{n+1}+Y_{n+1}\right] \\
& =\int_{0}^{t} h_{n}(x) \int_{0}^{t-x} \int_{0}^{v} h_{X}(u) h_{Y}(v-u) d u \bar{H}_{Z}(t-x \\
& -v) d v d x
\end{aligned}
$$

The selling time of the three products is based on either the season starts or when $\mathrm{k}$ triplets are produced $T=$ $\min ($ time to produce $\mathrm{k}$ triplets, the time from which the season starts).

The p.d.f of $T$

$$
\begin{aligned}
f_{T}(t) & =h_{k}(t) e^{-\lambda t}+\lambda e^{-\lambda t} \sum_{i=0}^{k-1} \int_{0}^{t} h_{i}(x) \bar{H}_{X}(t-x) d x \\
& +\lambda e^{-\lambda t} \sum_{i=0}^{k-1} \int_{0}^{t} h_{i}(x) \int_{0}^{t-x} h_{X}(u) \bar{H}_{Y}(t-x-u) d u d x \\
& +\lambda e^{-\lambda t} \sum_{i=0}^{k-1} \int_{0}^{t} h_{i}(x) \int_{0}^{t-x} \int_{0}^{v} h_{X}(u) h_{Y}(v-u) \bar{H}_{Z}(t-x-v) d u d v d x
\end{aligned}
$$

An explanation of the four terms in the right hand side of Equation 3.3 will be in order. As regards the first term, it provides the probability distribution function for the time $t$ required for the production of $\mathrm{k}$ number of triplets while the season remains non-started up to time t. In the case of the second term, it indicates the probability distribution for the start of the season at time $t$, the time $x$ needed for the manufacture of $i$ number of the combined units of $A, B$ and $C$, while the $(i+1)^{\text {th }}$ product $A$ is not ready during the time $t-x$. Concerning the term, it referees to the probability distribution for the start of the season at time $t$, the time $\mathrm{x}$ to manufacture $i$ units of the combination of $A, B$ and $C$, the $(i+1)^{\text {th }}$ A product is manufactured at time $x+u$ but $(i+1)^{\text {th }} B$ product is not manufactured during the time $t-x-u$ with the restriction on $i$ provided by $0 \leq i \leq k-1$. Finally, the fourth term denotes the probability distribution for the start of the season at time $t$, the time $x$ to manufacture $i$ number of the combinations of $A, B$ and $C$, the $(i+1)^{\text {th }}$ product $A$ is manufactured at time $x+u$, the $(i+1)^{\text {th }} B$ product is manufactured at time $x+v$ but the product $C$ is not manufactured during the time $t-x-v$ with the constraint $0 \leq i \leq k-1$.

This states the joint p.d.f of time to begin sales $T$ and total sales duration $S$ is follows considering the sales duration of the triplet units in the first and second term; the sales time of triplet units and the sale time of the product $A$ in the third term; and the sales time of triplet units and the sales time of the products $A$ and $B$ in the fourth term.

$$
\begin{aligned}
f(x, y)= & h_{k}(x) e^{-\lambda x} s_{k}(y)+\lambda e^{-\lambda x} \sum_{i=0}^{k-1} \int_{0}^{x} h_{i}(u) \bar{H}_{X}(x-u) d u s_{i}(y) \\
+ & \lambda e^{-\lambda x} \sum_{i=0}^{k-1} \int_{0}^{x} h_{i}(u) \int_{0}^{x-u} h_{X}(v) \bar{H}_{Y}(x-u-v) d v d u \\
& +\lambda e^{-\lambda x} \sum_{i=0}^{k-1} \int_{0}^{x} s_{i}(p) s_{A}(y) \int_{0}^{v} h_{X}(w) h_{Y}(v-w) d w \bar{H}_{Z}(x-u-w) d v d u \\
& \times \int_{0}^{y} s_{i}(p) \int_{0}^{y-p} s_{A}(q) s_{B}(y-p-q) d p d q
\end{aligned}
$$

Here $s_{j}(y)$ is the $\mathrm{j}$ fold convolution of $s(y)$ with itself. The double Laplace transform of $(T, S)$

$$
\begin{aligned}
& f_{T, S}^{*}(\varepsilon, \eta)=\int_{0}^{\infty} \int_{0}^{\infty} e^{-\varepsilon x} e^{-\eta y} f_{T, S}(x, y) d x d y \\
& =\int_{0}^{\infty} \int_{0}^{\infty} e^{-\varepsilon x} e^{-\eta y} h_{k}(x) e^{-\lambda x} s_{k}(y) d x d y \\
& +\int_{0}^{\infty} \int_{0}^{\infty} e^{-\varepsilon x} e^{-\eta y} \lambda e^{-\lambda x} \sum_{i=0}^{k-1} \int_{0}^{x} h_{i}(u) \bar{H}_{X}(x-u) s_{i}(y) d u d x d y \\
& +\int_{0}^{\infty} \int_{0}^{\infty} e^{-\varepsilon x} e^{-\eta y} \lambda e^{-\lambda x} \sum_{i=0}^{k-1} \int_{0}^{x} h_{i}(u) \int_{0}^{x-u} h_{X}(v) \bar{H}_{Y}(x-u-v) d v d u \int_{0}^{y} s_{i}(p) s_{A}(y-q) d p d x d y \\
& +\int_{0}^{\infty} \int_{0}^{-\varepsilon x} e^{-\eta y} \lambda e^{-\lambda x} \sum_{i=0}^{k-1} \int_{0}^{x} h_{i}(u) \int_{0}^{x-u} \int_{0}^{v} h_{X}(w) h_{Y}(v-w) d w \bar{H}_{Z}(x-u-v) d v d u \\
& \times \int_{0}^{y} s_{i}(p) \int_{0}^{y-p} s_{A}(q) s_{B}(y-p-q) d p d q d x d y \\
& f_{T, S}^{*}(\varepsilon, \eta)=h^{* *}(\varepsilon+\lambda) s^{* *}(\eta)+\lambda \sum_{i=0}^{k-1} h^{*}(\varepsilon+\lambda) \bar{H}_{X}^{*}(\varepsilon+\lambda) s^{* i}(\eta) \\
& +\lambda \sum_{i=0}^{k-1} h^{*}(\varepsilon+\lambda) h_{X}^{*}(\varepsilon+\lambda) \bar{H}_{Y}^{*}(\varepsilon+\lambda) s^{*}(\eta) s_{A}(\eta) \\
& +\lambda \sum_{i=0}^{k-1} h^{* i}(\varepsilon+\lambda) h_{X}^{*}(\varepsilon+\lambda) h_{Y}^{*}(\varepsilon+\lambda) \bar{H}_{Y}^{*}(\varepsilon+\lambda) s^{*}(\eta) s_{A}(\eta) s_{B}(\eta)
\end{aligned}
$$

This gives on simplification

$$
\begin{aligned}
f^{*}(\varepsilon, \eta) & =h^{* *^{*}}(\varepsilon+\lambda) s^{* *}(\eta)+\lambda\left[\frac{1-\left(h^{*}(\varepsilon+\lambda) s^{*}(\eta)\right)^{k}}{1-\left(h^{*}(\varepsilon+\lambda) s^{*}(\eta)\right)}\right] \bar{H}_{X}^{*}(\varepsilon+\lambda) \\
& +\lambda\left[\frac{1-\left(h^{*}(\varepsilon+\lambda) s^{*}(\eta)\right)^{k}}{1-\left(h^{*}(\varepsilon+\lambda) s^{*}(\eta)\right)}\right] h_{X}^{*}(\varepsilon+\lambda) \bar{H}_{Y}^{*}(\varepsilon+\lambda) s_{A}^{*}(\eta) \\
& +\lambda\left[\frac{1-\left(h^{*}(\varepsilon+\lambda) s^{*}(\eta)\right)^{k}}{1-\left(h^{*}(\varepsilon+\lambda) s^{*}(\eta)\right)}\right] h_{X}^{*}(\varepsilon+\lambda) h_{Y}^{*}(\varepsilon+\lambda) \bar{H}_{Z}^{*}(\varepsilon+\lambda) s_{A}^{*}(\eta) s_{B}^{*}(\eta)
\end{aligned}
$$

The Laplace transform of $\mathrm{T}$ is

$$
\begin{aligned}
f^{*}(\varepsilon, 0) & =h^{* *}(\varepsilon+\lambda)+\lambda\left[\frac{1-\left(h^{*}(\varepsilon+\lambda)\right)^{k}}{1-\left(h^{*}(\varepsilon+\lambda)\right)}\right] \bar{H}_{X}^{*}(\varepsilon+\lambda) \\
& +\lambda\left[\frac{1-\left(h^{*}(\varepsilon+\lambda)\right)^{k}}{1-\left(h^{*}(\varepsilon+\lambda)\right)}\right] h_{X}^{*}(\varepsilon+\lambda) \bar{H}_{Y}^{*}(\varepsilon+\lambda) \\
& +\lambda\left[\frac{1-\left(h^{*}(\varepsilon+\lambda)\right)^{k}}{1-\left(h^{*}(\varepsilon+\lambda)\right)}\right] h_{X}^{*}(\varepsilon+\lambda) h_{Y}^{*}(\varepsilon+\lambda) \bar{H}_{Z}^{*}(\varepsilon+\lambda)
\end{aligned}
$$

On differentiation of Equation 3.7, $\frac{\partial}{\partial \varepsilon} f^{*}(0,0)=-E(T)$

Published By: 


$$
\begin{aligned}
E(T)= & -k h^{* k-1}(\lambda) h^{*}(\lambda)+\lambda\left[\frac{k h^{* k-1}(\lambda) h^{*}(\lambda)}{1-h^{*}(\lambda)}\right] \bar{H}_{X}^{*}(\lambda) \\
& -\lambda\left[\frac{1-\left(h^{*}(\lambda)\right)^{k}}{\left(1-h^{*}(\lambda)\right)^{2}}\right] h^{*}(\lambda)\left[\bar{H}_{X}^{*}(\lambda)\right] \\
& -\lambda\left[\frac{1-\left(h^{*}(\lambda)\right)^{k}}{\left(1-h^{*}(\lambda)\right)}\right] \bar{H}_{X}^{*}(\lambda) \\
& +\lambda\left[\frac{k h^{* k-1}(\lambda) h^{*}(\lambda)}{1-h^{*}(\lambda)}\right]\left[h_{X}^{*}(\lambda) \bar{H}_{Y}^{*}(\lambda)\right] \\
& -\lambda\left[\frac{\left.1-h^{*}(\lambda)\right)^{k}}{\left(1-h^{*}(\lambda)\right)^{2}}\right] h^{*}(\lambda)\left[h_{X}^{*}(\lambda) \bar{H}_{Y}^{*}(\lambda)\right] \\
& -\lambda\left[\frac{1-\left(h^{*}(\lambda)\right)^{k}}{\left(1-h^{*}(\lambda)\right)}\right]\left\{h_{X}^{*}(\lambda) \bar{H}_{Y}^{*}(\lambda)+h_{X}^{*}(\lambda) \bar{H}_{Y}^{*}(\lambda)\right\} \\
& +\lambda\left[\frac{k h^{* k-1}(\lambda) h^{*}(\lambda)}{1-h^{*}(\lambda)}\right]\left\{h_{X}^{*}(\lambda) h_{Y}^{*}(\lambda) \bar{H}_{Z}^{*}(\lambda)\right\} \\
& -\lambda\left[\frac{1-\left(h^{*}(\lambda)\right)^{k}}{\left(1-h^{*}(\lambda)\right)^{2}}\right] h^{*}(\lambda)\left\{h_{X}^{*}(\lambda) h_{Y}^{*}(\lambda) \bar{H}_{Z}^{*}(\lambda)\right\} \\
& -\lambda\left[\frac{1-\left(h^{*}(\lambda)\right)^{k}}{\left(1-h^{*}(\lambda)\right)}\right]\left\{h_{X}^{*}(\lambda) h_{Y}^{*}(\lambda) \bar{H}_{Y}^{*}(\lambda)+h_{X}^{*}(\lambda) h_{Y}^{*}(\lambda) \bar{H}_{Z}^{*}(\lambda)\right\}
\end{aligned}
$$

Hence the expected time

$$
E(T)=\frac{1-\left(h^{*}(\lambda)\right)^{k}}{\lambda}
$$

The Laplace transform of total sales time $\mathrm{S}$ is

$$
\begin{aligned}
f^{*}(0, \eta) & =h^{*}(\lambda) s^{*}(\eta)+\lambda\left[\frac{1-\left(h^{*}(\lambda) s^{*}(\eta)\right)^{k}}{1-\left(h^{*}(\lambda) s^{*}(\eta)\right)}\right] \bar{H}_{X}^{*}(\lambda) \\
& +\lambda\left[\frac{1-\left(h^{*}(\lambda) s^{*}(\eta)\right)^{k}}{1-\left(h^{*}(\lambda) s^{*}(\eta)\right)}\right] h_{X}^{*}(\lambda) \bar{H}_{Y}^{*}(\lambda) s_{A}^{*}(\eta) \\
& +\lambda\left[\frac{1-\left(h^{*}(\lambda) s^{*}(\eta)\right)^{k}}{1-\left(h^{*}(\lambda) s^{*}(\eta)\right)}\right] h_{X}^{*}(\lambda) h_{Y}^{*}(\lambda) \bar{H}_{Z}^{*}(\lambda) s_{A}^{*}(\eta) s_{B}^{*}(\eta)
\end{aligned}
$$

Similarly $\frac{\partial}{\partial \eta} f^{*}(0,0)=-E(S)$

$$
\begin{aligned}
& E(S)=-h^{h^{*}}(\lambda) k(-E(S))-\lambda\left[\frac{k h^{* *}(\lambda) E\left(s_{1}\right)}{1-h^{* *}(\lambda)}\right] \bar{H}_{X}^{*}(\lambda) \\
&-\lambda\left[\frac{1-h^{* *}(\lambda)}{\left(1-h^{*}(\lambda)\right)^{2}}\right] h^{*}(\lambda)\left(-E\left(s_{1}\right)\right) \bar{H}_{X}^{*}(\lambda) \\
&-\lambda\left[\frac{k h^{* *}(\lambda) E\left(s_{1}\right)}{1-h^{* *}(\lambda)}\right]\left\{h_{X}^{*}(\lambda) \bar{H}_{Y}^{*}(\lambda)\right\} \\
&-\lambda\left[\frac{1-h^{* *}(\lambda)}{\left(1-h^{*}(\lambda)\right)^{2}}\right] h^{* *}(\lambda)\left(-E\left(s_{1}\right)\right)\left\{h_{X}^{*}(\lambda) \bar{H}_{Y}^{*}(\lambda)\right\} \\
&-\lambda\left[\frac{1-h^{* *}(\lambda)}{\left(1-h^{*}(\lambda)\right)}\right]\left\{h_{X}^{*}(\lambda) \bar{H}_{Y}^{*}(\lambda)\right\}\left(-E\left(s_{A}\right)\right) \\
&-\lambda\left[\frac{k h^{* *}(\lambda) E\left(s_{1}\right)}{1-h^{* *}(\lambda)}\right]\left\{h_{X}^{*}(\lambda) h_{Y}^{*}(\lambda) \bar{H}_{Z}^{*}(\lambda)\right\} \\
&-\lambda\left[\frac{1-h^{* *}(\lambda)}{\left(1-h^{*}(\lambda)\right)^{2}}\right] h^{*}(\lambda)\left(-E\left(s_{1}\right)\right)\left\{h_{X}^{*}(\lambda) h_{Y}^{*}(\lambda) \bar{H}_{Z}^{*}(\lambda)\right\} \\
&-\lambda\left[\frac{1-h^{* *}(\lambda)}{\left(1-h^{*}(\lambda)\right)}\right]\left\{h_{X}^{*}(\lambda) h_{Y}^{*}(\lambda) \bar{H}_{Z}^{*}(\lambda)\right\}\left(-E\left(s_{A}\right)\right) \\
&-\lambda\left[\frac{1-h^{* *}(\lambda)}{\left(1-h^{*}(\lambda)\right)}\right]\left\{h_{X}^{*}(\lambda) h_{Y}^{*}(\lambda) \bar{H}_{Z}^{*}(\lambda)\right\}\left(-E\left(s_{B}\right)\right) \\
& E(S)=\left[\frac{1-h^{*}(\lambda)}{1-h^{*}(\lambda)}\right]\left\{h^{*}(\lambda)\left[E\left(s_{1}\right)-E\left(s_{A}\right)-E\left(s_{B}\right)+E\left(s_{A}\right) h_{X}^{*}(\lambda)\right]\right\} \\
&+E\left(s_{B}\right) h_{X}^{*}(\lambda) h_{Y}^{*}(\lambda)
\end{aligned}
$$

\section{SPECIAL CASES}

Most of stochastic models, the c.d.f are exponential. In the Equation 3.12, $\mathrm{k}$ is constant and sales for the system are considered when it completes the production of $\mathrm{k}$ triplets. As a more general case, $\mathrm{k}$ may be treated as a random variable taking positive integral values such that

$$
P(k=i)=p_{i}>0, i>0 \text { and } \sum_{i=1}^{n} p_{i}=1 \text {. }
$$

The unique case in which $\mathrm{X}$ and $\mathrm{Y}$ are exponential random variables with parameters $1, \mathrm{~m}$ and $\mathrm{n}$ respectively is considered below which states

$$
\begin{gathered}
h^{*}{ }_{X}(\lambda)=\frac{l}{l+\lambda} ; h^{*^{\prime}}{ }_{X}(\lambda)=\frac{-l}{(l+\lambda)^{2}} \\
h^{*}{ }_{Y}(\lambda)=\frac{m}{m+\lambda} ; h^{*^{\prime}}{ }_{Y}(\lambda)=\frac{-m}{(m+\lambda)^{2}} \\
h^{*}{ }_{Z}(\lambda)=\frac{n}{n+\lambda} \quad ; h^{*^{\prime}}{ }_{Z}(\lambda)=\frac{-n}{(n+\lambda)^{2}} \\
\bar{H}_{X}{ }_{X}(\lambda)=\frac{1}{l+\lambda} \quad ; \bar{H}^{*^{\prime}}{ }_{X}(\lambda)=\frac{-1}{(l+\lambda)^{2}} \\
\bar{H}^{*}{ }_{Y}(\lambda)=\frac{1}{m+\lambda} ; \bar{H}^{*^{\prime}}{ }_{Y}(\lambda)=\frac{-1}{(m+\lambda)^{2}} \\
\bar{H}^{*}{ }_{Z}(\lambda)=\frac{1}{n+\lambda} ; \quad \bar{H}^{*^{\prime}}{ }_{Z}(\lambda)=\frac{-1}{(n+\lambda)^{2}}
\end{gathered}
$$

Using Equation 3.10 to 3.13, E (T) and E (S) are found when $\mathrm{X}$ and $\mathrm{Y}$ are exponentials with parameter $1, \mathrm{~m}$ and $\mathrm{n}$ as follows.

$$
\begin{aligned}
& E(T)=\left[1-\left(\frac{l m n}{(l+\lambda)(m+\lambda)(n+\lambda)}\right)^{k}\right]\left(\frac{1}{\lambda}\right) \\
& E(S)=\left\{\frac{1-\left(\frac{l m n}{(l+\lambda)(m+\lambda)(n+\lambda)}\right)^{k}}{1-\left(\frac{l m n}{(l+\lambda)(m+\lambda)(n+\lambda)}\right)}\right\}\left[\left(\frac{l m n}{(l+\lambda)(m+\lambda)(n+\lambda)}\right)\right] \\
& \left(E\left(s_{1}\right)-E\left(s_{A}\right)-E\left(s_{B}\right)+E\left(s_{A}\right) \frac{l}{l+\lambda}+E\left(s_{B}\right) \frac{l}{l+\lambda} \frac{m}{m+\lambda}\right)
\end{aligned}
$$

\section{NUMERICAL EXAMPLE}

To demonstrate the applications in the Equations 3.14 and 3.15 different values for $1=1, \mathrm{~m}=2, \mathrm{n}=3, \lambda=4, \mathrm{E}\left(S_{1}\right)=$ $30, E\left(S_{A}\right)=10$ and $E\left(S_{B}\right)=20$.

Graphs of $E(T)$ and $E(S)$ for Different values of $k$

Using the Equation 3.13 and 3.14 are found and the corresponding graphs are drawn.

Table 3.1: Numerical tabulation for obtaining of $E$ (T) and $E(S)$ values

\begin{tabular}{|c|c|c|c|c|c|c|c|c|}
\hline $\mathbf{K}$ & $\mathbf{5}$ & $\mathbf{6}$ & $\mathbf{7}$ & $\mathbf{8}$ & $\mathbf{9}$ & $\mathbf{1 0}$ & $\mathbf{1 1}$ & $\mathbf{1 2}$ \\
\hline $\mathrm{E}(\mathrm{T})$ & 0.249 & 0.249 & 0.249 & 0.249 & 0.249 & 0.249 & 0.249 & 0.250 \\
\hline $\mathrm{E}(\mathrm{S})$ & 3.429 & 3.527 & 3.529 & 3.529 & 3.529 & 3.529 & 3.529 & 3.529 \\
\hline
\end{tabular}




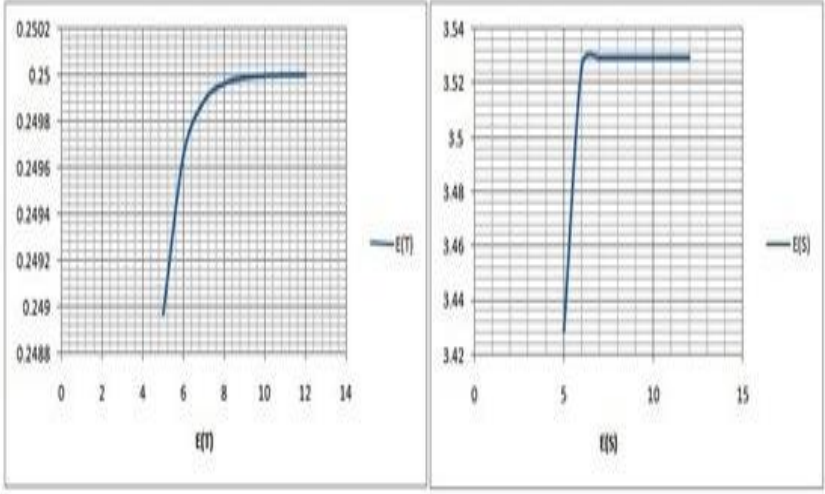

Figure 3.1: Graphs of E (T) and E (S)

Inference: From the table 3.1, when the value of $k$ increases, $\mathrm{E}(\mathrm{T})$ and $\mathrm{E}(\mathrm{S})$ also increases.

Graphs of $\mathbf{E}(\mathbf{T})$ and $E(S)$

Taking $1=1, \lambda=1$ to $6, \mathrm{E}\left(S_{1}\right)=20$ and $\mathrm{k}=1$ to 6 , Graphs of $\mathrm{E}(\mathrm{T})$ and $\mathrm{E}(\mathrm{S})$ are drawn.

Table 3.2: Numerical tabulation for obtaining of $\mathbf{E}(\mathbf{T})$ values

\begin{tabular}{|c|c|c|c|c|c|c|}
\hline & \multicolumn{5}{|c|}{$\mathrm{E}(\mathrm{T})$} \\
\hline $\mathrm{K}$ & $\lambda=1$ & $\lambda=2$ & $\lambda=3$ & $\lambda=4$ & $\lambda=5$ & $\lambda=6$ \\
\hline 1 & 0.75 & 0.45 & 0.317 & 0.243 & 0.196 & 0.165 \\
\hline 2 & 0.938 & 0.495 & 0.319 & 0.245 & 0.198 & 0.166 \\
\hline 3 & 0.984 & 0.499 & 0.333 & 0.250 & 0.200 & 0.167 \\
\hline 4 & 0.996 & 0.499 & 0.333 & 0.250 & 0.200 & 0.167 \\
\hline 5 & 0.996 & 0.500 & 0.333 & 0.250 & 0.200 & 0.167 \\
\hline 6 & 0.999 & 0.500 & 0.3333 & 0.250 & 0.200 & 0.167 \\
\hline
\end{tabular}

Table 3.3: Numerical tabulation for obtaining of $\mathbf{E}(\mathrm{S})$ values

\begin{tabular}{|c|c|c|c|c|c|c|}
\hline & \multicolumn{5}{|c|}{$\mathrm{E}(\mathrm{S})$} \\
\hline $\mathrm{K}$ & $\lambda=1$ & $\lambda=2$ & $\lambda=3$ & $\lambda=4$ & $\lambda=5$ & $\lambda=6$ \\
\hline 1 & 11.667 & 6.6667 & 4.5 & 3.3333 & 2.619 & 2.1429 \\
\hline 2 & 14.5833 & 7.3333 & 4.725 & 3.4286 & 2.6658 & 2.1684 \\
\hline 3 & 15.3125 & 7.4 & 4.7363 & 3.4313 & 2.6667 & 2.1687 \\
\hline 4 & 15.4948 & 7.4067 & 4.7368 & 3.4314 & 2.6667 & 2.1687 \\
\hline 5 & 15.5404 & 7.4073 & 4.7368 & 3.4314 & 2.6667 & 2.1687 \\
\hline 6 & 15.5518 & 7.4074 & 4.7368 & 3.4314 & 2.6667 & 2.1687 \\
\hline
\end{tabular}

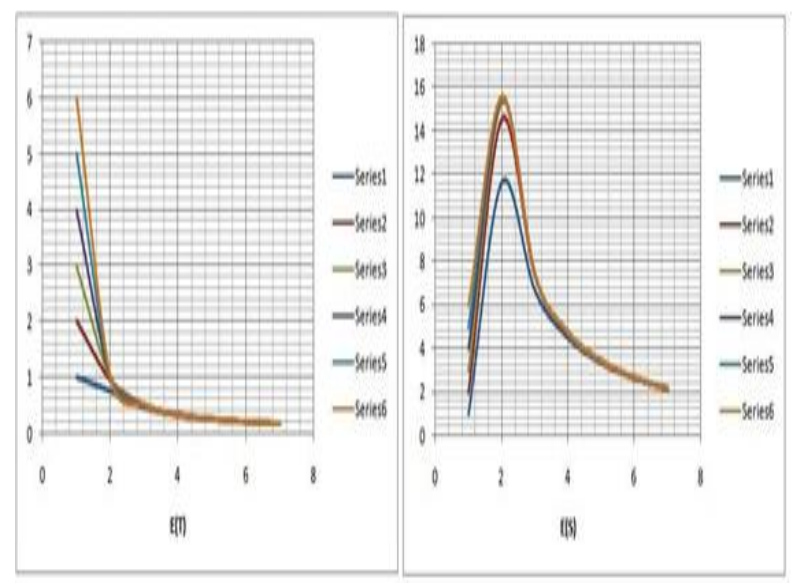

Figure 3.2: Graphs of $E(T)$ and $E(S)$
Inference: From the table 3.2 and 3.3 , as $\mathrm{k}$ increases and the parameter $\lambda$ is fixed, the expected values of $E(T)$ and $E$ (S) increases. As $\mathrm{k}$ is fixed and the parameter $\lambda$ increases, the expected values of E (T) and E (S) decreases.

\section{CONCLUSION}

When $\mathrm{k}$, the number of products increases, both the expected time for sales $\mathrm{E}(\mathrm{T})$ and sales time $\mathrm{E}(\mathrm{S})$ increases.

When the exponential parameter $\lambda$ increases, the expected time for sales E (T) decreases and sales time E (S) increases.

As $\mathrm{k}$ increases and the parameter $\lambda$ are fixed, the expected values $E(T)$ and $E(S)$ increases. As $k$ is fixed and the parameter $\lambda$ increases, the expected value $\mathrm{E}(\mathrm{T})$ and $\mathrm{E}$ (S) decreases.

\section{REFERENCES}

1. H. Aydi, "Some coupled fixed point results on partial metric spaces", International Journal of Mathematical Sciences, Artical ID 647091, 2011..

2. H. Aydi, "Some fixed point results in ordered partial metric spaces", arXiv:1103.3680v1(math.GN), 2011

3. T.G. Bhaskar and V. Lakshmikantham, "Fixed point theorems in partially ordered metric spaces and applications", Nonlinear Analysis: Theory, Methods and Apllications, 65(7), 13791393, 2006.

4. L.Ciric and V. Lakshmikantham, "Coupled random fixed point theorems for nonlinear contractions in partially ordered metric spaces", Stochastic and Applications, 27, 1246-1259, 2009.

5. L.Ciric and V. Lakshmikantham, "Coupled fixed point theorems for nonlinear contractions in partially ordered metric spaces", Nonlinear Analysis: Theory, Methods and Applications 70 (12) , 4341-4349, 2009.

6. L.Ciric, M.O. Olatinwo, D. Gopal, G. Akinbo, "Coupled fixed point theorems for mappings satisfing a contractions of rational type on a partially ordered metric space", Advances in Fixed Point Theory, 2 (1), 1-8, 2012.

7. S.G. Matthews, "Partial metric topology, in: Proc 8th summer conference on general topology and application", in Annals of the New York Academy of Sciences, 728, 183-197, 1994.

8. J. G. Mehta and M.L.Joshi, "On coupled fixed point theorem in partially ordered complete metric space", Int. J. Pure Appl. Sci. Technol.,1(2), 87-92, 2010.

9. S. J. O'Neill, "Two topologies are better than one", Tech. Rep., University of warwick, Coventry, UK 1995.

10. S. J. O'Neill, "Partial metrics, valuations and domain theory, in: Proc 11th summer conference on general topology and application", in Annals of the New York Academy of Sciences, 806, 304-315, 1996.

11.A. C. M. Ran, M. C. B. Reurings, "A fixed point theorem in partially ordered sets and some applications to matrix equations", Proc. Amer. Math. Soc. 132, 1435-1443, 2004. 